\title{
Transovarial Transmission of Dengue Virus in Aedes aegypti: A Case in Quezon City, Philippines
}

\author{
Ralph Julius G. Bawalan¹ Nelia P. Salazar² and Francisco M. Heralde III ${ }^{3}$ \\ ${ }^{1}$ The Graduate School, University of the East Ramon Magsaysay Memorial Medical Center, Inc., Quezon City, Philippines \\ ${ }^{2}$ Research Institute for Tropical Medicine, Department of Health, Muntinlupa City, Philippines \\ ${ }^{3}$ Department of Biochemistry and Molecular Biology, College of Medicine, University of the Philippines Manila
}

\begin{abstract}
Background. The changing nature of dengue epidemiology and control makes dengue one of the challenging infectious disease problems in the present time with certain inadequacies in existing knowledge base becoming apparent.

Objective. This quantitative and experimental study was conducted to provide recent local evidence that dengue virus transovarial transmission among field collected Aedes aegypti mosquitoes does occur and presents an important factor in the epidemiology and control of dengue.

Methods. Households in Quezon City, Philippines, a known dengue infection hotspot in 2011, were randomly selected (H9 and $\mathrm{H14}$ ) for Aedes aegypti egg and larval collection. Mosquito larvae were captured using standard ovitraps and reared to adulthood in the entomology unit of the Molecular Diagnostics and Genotyping Laboratory at the University of the Philippines (UP), College of Medicine, Manila. Whole organism homogenate of adult mosquitoes were prepared for subsequent dengue virus molecular characterization and virulence testing. Both egg samples and adult Aedes mosquitoes were processed as pooled samples and their infection profile for dengue virus was determined by serotype specific RT-PCR.
\end{abstract}

Results. Molecular test results show that in each household and in each generation (parent, F1 and F2), there were detectable and strong dengue viral presence, predominantly the serotypes DEN-2, DEN-3 and DEN-4 in the Aedes aegypti mosquito homogenates.

Conclusions. These laboratory evidences indicate that transovarial transmission of dengue virus does occur in a high urban city like Quezon City where incidence of dengue is high.

\footnotetext{
Paper presented at the16th Annual Research Forum of the Research Institute for the Health Sciences, February 13, 2014, UERM Quezon City; 26th Annual Scientific Conference of the Philippine Association for Laboratory Animal Science, May 22, 2014, Rembrandt Hotel, Quezon City; and 41st Annual Scientific Conference of the Philippine Society of Biochemistry and Molecular Biology, December 4-5, 2014, Marco Polo Hotel, Cebu City.

Corresponding author: Ralph Julius G. Bawalan, MSTM

The Graduate School

University of the East Ramon Magsaysay Memorial Medical Center, Inc.

Quezon City, Philippines

Email: ralph.bawalan@gmail.com
}

Thus, it is important to consider the existence of this phenomenon in existing and future dengue control programs to ensure effectiveness of community-based intervention strategies.

Key Words: Aedes aegypti, transovarial transmission, dengue PCR, serotype-specific RT-PCR, community-based intervention

\section{Introduction}

Transovarial transmission, or vertical transmission of the dengue virus, is an etiologic phenomenon responsible for the persistence of the virus during inter-epidemic periods. ${ }^{1}$ While majority of dengue viruses typically are transmitted from vector to vertebrate host cycle, there are in vivo and in vitro evidences of transmission through the vertical route where the parent mosquito is able to transfer the virus to the next generation, ${ }^{2-5}$ and where the larval offspring are shown to typically contain the same virus particles as the parent. In fact, evidence of vertical transmission up to the seventh generation in laboratory-infected models were demonstrated in two studies. 6,7

The changing nature of dengue epidemiology and how dengue virus is maintained even during inter-epidemic periods raised questions that led to different researches evaluating the importance of transovarial transmission of dengue virus in the wild stock. ${ }^{5}$ Factoring in of vertical transmission in the equation of dengue epidemiology would significantly impact the community-based intervention currently practiced and those that will be designed in the future. The Philippines is one of the countries that suffers substantially from dengue epidemics; however, there has been limited studies related to the dynamics of inter and intra-transmission of dengue virus in mosquitos particularly those geared towards understanding dengue pathogenesis.

Quezon City, Philippines, in particular, has been declared by the health department as an urban dengue hotspot in 2011; hence, it would be interesting to investigate whether transovarial transmission of the dengue virus by Aedes aegypti plays a role in the persistent presence of the virus in the area, and whether it might be the potential origin of the continuous introduction of the dengue virus during epidemic periods. This study aimed to determine and describe evidences of transovarial transmission of dengue 
virus from field collected Aedes aegypti to understand the dynamics of viral persistence in the wild stock as part of the project geared towards development of community-based intervention and control.

\section{Methods}

\section{Study Site and Field Collection}

Quezon City is a high urban, residential area in the National Capital Region. Based on the 2011 Disease Surveillance report of the Department of Health, ${ }^{10}$ there were 1261 dengue cases and 13 dengue deaths in the city. Among its barangays, Barangay Old Balara, with population of 71,220 (NSO, 2010) was selected as a project experimental site, with it being one of the barangays that posted highest increase in dengue cases. Of the houses in the area, two households were selected through random sampling and located by global positioning system (GPS) locators.

Field egg and larval Aedes aegypti samples from Quezon City (endemic Philippine strain), coming from households

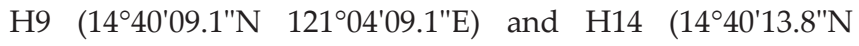
$\left.121^{\circ} 04^{\prime} 09.1^{\prime \prime E}\right)$ in Barangay Old Balara, Area Luzon, Quezon City, were collected using Ovicidal and Larvicidal (OL) mosquito traps based on Mistica et al model,12 and larval samples were reared in the laboratory using containers with tight lids to prevent escape, as these samples were grown to adults and used for viral analysis.

\section{Mosquito Identification}

Collected eggs were counted and identified by epidemiologic week, and hatched into larvae until they mature to adults. Another set of mosquito eggs were separated for viral detection. The adult mosquitoes were submitted to the Research Institute for Tropical Medicine (RITM) Medical Entomology Laboratory for taxonomic identification through microscopic observation of the morphology of abdominal segments, palps, wing scales, and proboscis and were verified molecularly through their mtCO1 sequences following the procedures of Chen et al. ${ }^{13}$ and reported by Heralde. ${ }^{14}$

\section{Dengue Virus Isolation and Viremia Detection}

Detection of dengue virus in adult and egg mosquitoes were performed with serotype specific primers (Table 1) through reverse transcriptase-polymerase chain reaction (RT-PCR) following the protocol of Lanciotti et al. ${ }^{8}$ Mosquito pools ( $\mathrm{n}=10$ for mosquito eggs; $\mathrm{n}=10$ for mosquito adults for both $\mathrm{H} 9$ and H14) were homogenized using a sample disruptor and centrifuged at 40,000 rpm. Total RNA was extracted from the mosquito suspension using Trizol $\AA$ (Ambion, USA) following the manufacturer's instruction. Using the RNA extracts as template, first strand synthesis was initiated through reverse transcriptase. Five microliters (uL) of RNA in 50uL reaction volume were used with Qiagen OneStep RT PCR kit® (Qiagen, Netherlands). RTPCR was carried out at $55^{\circ} \mathrm{C}$ annealing temperature. The resultant PCR product was diluted in 1:100 in water. Nested RT-PCR of dengue virus RNA was then carried out using DENV consensus and serotype specific primers. Five (5) uL of diluted RT-PCR product was incorporated in a $50 \mathrm{uL}$ reaction volume of the TaqPCR Master Mix Kit ${ }^{\circledR}$ (Qiagen, Netherlands). Initial denaturation for three minutes at $94^{\circ} \mathrm{C}$ was followed by 25 cycles, each consisting of $94^{\circ} \mathrm{C}$ denaturation for 30 seconds, $50^{\circ} \mathrm{C}$ annealing for one minute, and $72^{\circ} \mathrm{C}$ extension for one minute, followed by a final extension step of $72^{\circ} \mathrm{C}$ for 10 minutes.

RT-PCR was performed to detect the virus in subsequent pooled mosquito adults and mosquito egg samples using standard PCR procedures with minimal modifications. Dengue serotypes were identified and detected as previously outlined. The presence of dengue virus was based on a conforming base pair bands after electrophoresis, wherein a positive result was exemplified by the presence of the correct size of band during electrophoresis visualized under ultraviolet light; and a negative result with the absence of bands. The Aedes samples from RITM that were negative for dengue were used as control. The collected field larval samples were reared in the laboratory using containers with tight lids to prevent escape, until these larvae develop into adults. Five (5) male and 5 female mosquitoes were then randomly selected from each location, and were allowed to lay eggs until they hatch. These samples were then reared for the next generation. The

Table 1. Primer and Probe sequences used for RT PCR for dengue virus serotype identification based on Lanciotti et al. ${ }^{8}$

\begin{tabular}{lll}
\hline Primer name and sequence & Location & Size in bp of amplified DNA product (primers) \\
\hline D1 5'-TCAATATGCTGAAACGCGCGAGAAACCG-3' & $134-161$ & 511 \\
D2 5'-TTGCACCAACAGTCAATGTCTTCAGGTTC-3' & $616-644$ & \\
TS1 5'-CGTCTCAGTGATCCGGGGG-3' & $568-586$ & 511 \\
TS2 5'-CGCCACAAGGGCCATGAACAG-3' & $232-252$ & \\
TS3 5'-TAACATCATCATGAGACAGAGC-3' & $400-421$ & 482 (Dl and TS1) \\
TS4 5'-CTCTGTTGTCTTAAACAAGAGA-3' & $506-527$ & \\
& & 119 (Dl and TS2) \\
& & 290 (Dl and TS3) \\
& & 392 (Dl and TS4) \\
\hline
\end{tabular}


F1 progeny were reared to adulthood and were retained for 5-7 days after emergence in order to guarantee mating. Pupae were collected and counted daily in order to prevent adult mosquito from emerging in the larval rearing container. Adults were contained in sturdy cages. Similar procedure was performed until F2 generation is produced.

Dengue serotypes (DEN1-4) were identified and detected for the 15 male and 15 female mosquitoes of the parent generation based on the expected amplicon sizes for each serotype. Only those that turned out positive for viral presence were continued for monitoring and were bred again for the succeeding generation. Those that turned negative were discarded except for one pair which was maintained as control. This procedure was repeated until the second generation (F2) of offspring was produced. PCR products were analyzed by gel electrophoresis using a $2 \%$ agarose gel (Vivantis, USA) containing Gel Red $(0.5 \mathrm{ug} / \mathrm{mL})$. A 100-bp ladder was used as a size standard. A band on the agarose gel of the correct size was interpreted as a positive result. A faint band of the correct size was considered an equivocal result while the absence of bands was interpreted as a negative result. Shaved ICR (CD-1®) mice, which were RT-PCR verified to be dengue negative were used as blood source for the female Aedes mosquitoes. This procedure was performed following the Institutional Animal Care and Use Committee (IACUC) guidelines ensuring the well-being, safety, maintenance, and disposal of the mouse samples. All experiments involving animals in this study were approved by the Ethics Review Committee of the University of the East-Ramon Magsaysay (UERM) Research Institute for Health Sciences.

\section{Results}

\section{Research Sample Characteristics}

A total of 135 parent Aedes aegypti larvae were collected from sites H9 and H14 in Quezon City. Another set of 30 Aedes aegypti eggs from RITM (R) were reared to become larvae, and were used as the negative control. Five males and five females (m:f ratio of 1:1) were randomly selected from each location and were reared to adulthood in the entomology unit $(n=30)$ of the Molecular Diagnostics and Genotyping Laboratory of UP Manila College of Medicine. These pairs were used for: (1) dengue serotype identification, and (2) breeding for the next generation (Figure 1).

\section{Diagnosis of Dengue Virus in mosquito samples}

RT-PCR test for dengue virus, DEN-1, DEN-2, DEN-3, and DEN-4 in each group (H9, H14, and R sites) and in each generation (Parent, F1, F2 mosquitoes) was performed and the agarose gel electropherogram of the PCR products are shown in Figures 2, 3 and 4. A positive result was demonstrated by the formation of bands in the range 100$500 \mathrm{bp}$, depending on the viral type. In Figure 2, the field- collected parental mosquito samples were bred to ensure F1 recovery and subsequently sacrificed for serotype-specific RT-PCR. Amplicon compatible with Dengue serotype 1 (i.e., TS1) is obtained as shown in lane 3; Dengue serotype 2 (i.e., TS2) compatibles are shown in lanes 5, 6 and 7; Dengue serotype 3 (i.e. TS3) compatibles are shown in lanes 11, 12 and 13; and Dengue serotype 4 (i.e. TS4) compatibles are shown in Lane 16 and 17. Note the smudged nature of some of the amplicons indicating either a small quantity of template or reduced template specificity. Note that some of the amplicons were not the ideal sizes based on Table 1. However, sequence analysis showed they were dengue sequences of different genotypes from the reference genotype. ${ }^{14}$

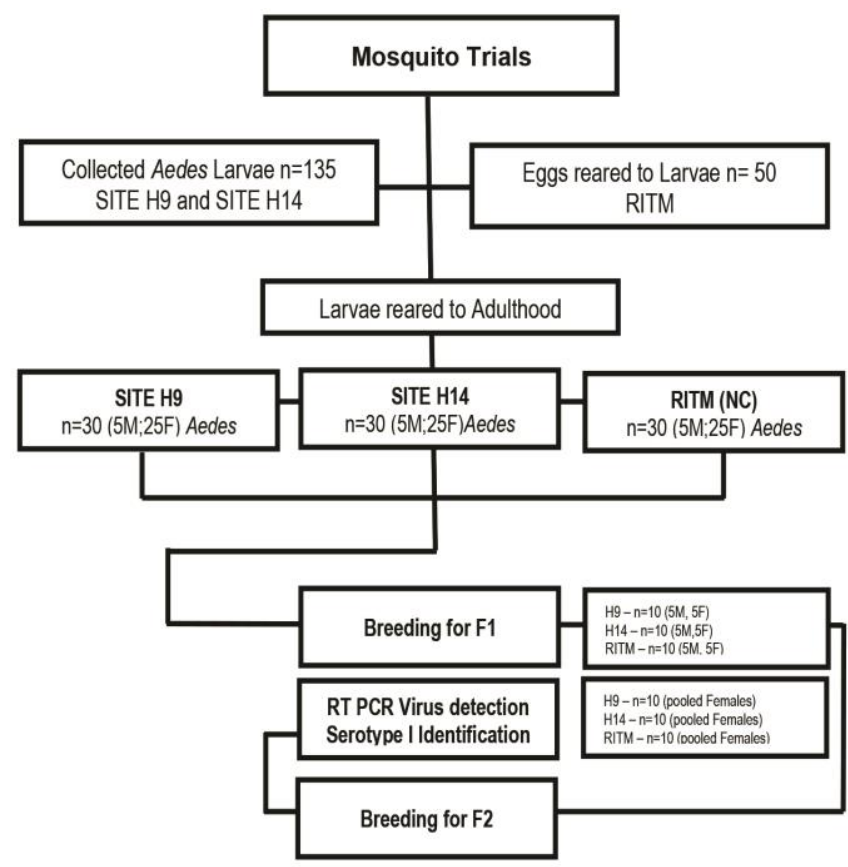

Figure 1. Experimental design implemented in this study to evaluate transovarial transmission of dengue virus in fieldcollected mosquitoes from Quezon City.

In Figure 3, F1 mosquitoes were bred to recover F2 generation, followed by subsequent homogenization for serotype-specific RT-PCR. Amplicons compatible with TS1 were obtained as shown in lane 2, 3 and 4; amplicon compatible with TS2 were shown in lanes 6 and 7; amplicon compatible with TS3 are shown in lanes 11, 12, and 13; and amplicon compatible with TS4 are shown in Lane 15, 16, and 17.

Figure 4 shows the serotype-specific profile of the F2 generation. It can be observed that amplicons compatible with TS1 are distinctly shown in lane 3 and 4; TS2 compatibles are shown in lanes 6, 7, and 8; TS3 compatibles are shown in lanes 11, 12, and 13; and TS4 compatibles are shown in Lane 15, 16, and 17. 


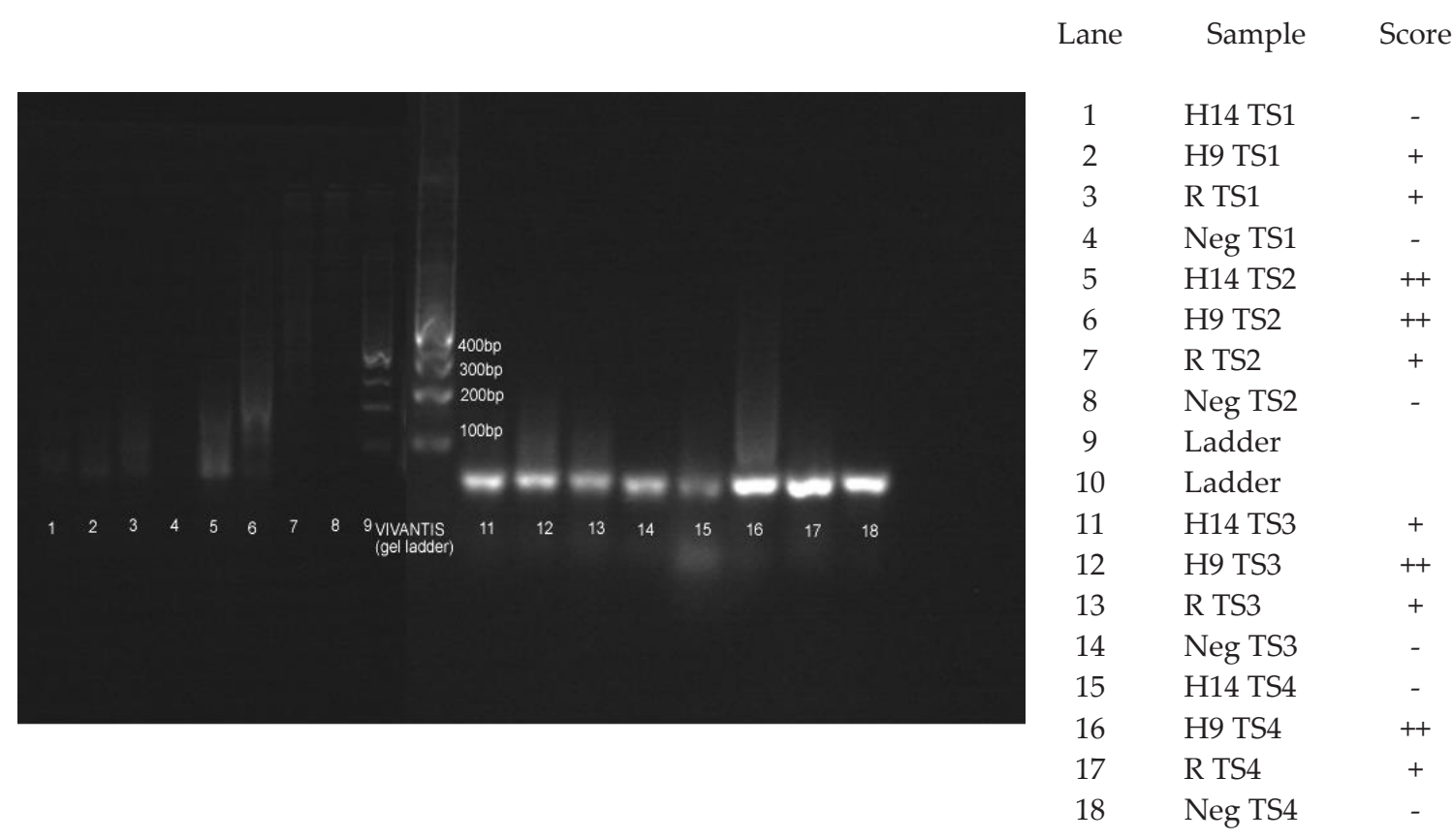

Figure 2. Electropherogram of dengue PCR amplicons from the parent generation of field-collected Aedes aegypti mosquitoes from H9, H14, RITM and Negative Control (Panel A) with their respective designations and scores shown in Panel B

\section{A. Electropherogram of dengue PCR amplicons}

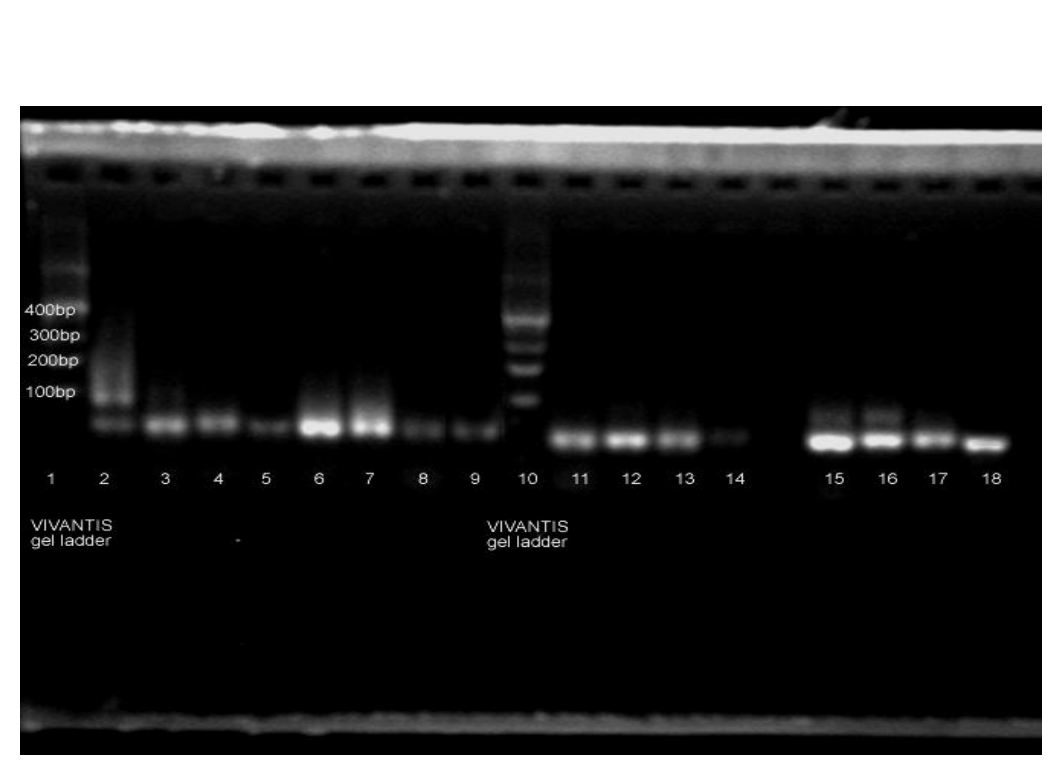

B. Designations

\begin{tabular}{clc} 
Lane & \multicolumn{1}{c}{ Sample } & Score \\
1 & Ladder & \\
2 & H14 TS1 & +++ \\
3 & H9 TS1 & + \\
4 & R TS1 & + \\
5 & Neg TS1 & - \\
6 & H14 TS2 & ++ \\
7 & H9 TS2 & ++ \\
8 & R TS2 & - \\
9 & Neg TS2 & - \\
10 & Ladder & \\
11 & H14 TS3 & + \\
12 & H9 TS3 & + \\
13 & R TS3 & + \\
14 & Neg TS3 & - \\
15 & H14 TS4 & ++ \\
16 & H9 TS4 & ++ \\
17 & R TS4 & + \\
18 & Neg TS4 & -
\end{tabular}

Figure 3. Electropherogram of dengue PCR amplicons from the F1 generation of Aedes aegypti mosquitoes from H9, H14, RITM and Negative Control (Panel A) with their respective designations and scores shown in Panel B. 


\section{A. Electropherogram of dengue PCR amplicons}

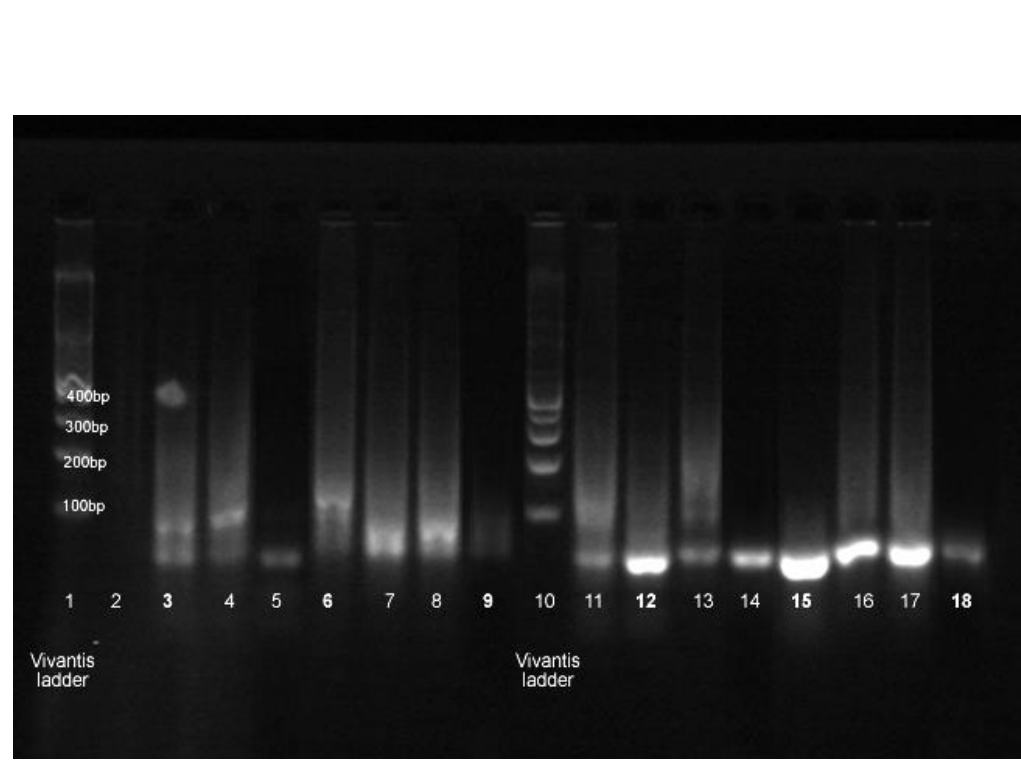

Figure 4. Electropherogram of dengue PCR amplicons of Aedes aegypti mosquitoes from H9, H14, RITM and with their respective designations and scores shown in Panel B.
B. Designations

Lane Sample Score

$\begin{array}{clc}1 & \text { Ladder } & \\ 2 & \text { H14 TS1 } & - \\ 3 & \text { H9 TS1 } & +++ \\ 4 & \text { R TS1 } & +++ \\ 5 & \text { Neg TS1 } & - \\ 6 & \text { H14 TS2 } & ++ \\ 7 & \text { H9 TS2 } & ++ \\ 8 & \text { R TS2 } & ++ \\ 9 & \text { Neg TS2 } & - \\ 10 & \text { Ladder } & \\ 11 & \text { H14 TS3 } & ++ \\ 12 & \text { H9 TS3 } & ++ \\ 13 & \text { R TS3 } & ++ \\ 14 & \text { Neg TS3 } & - \\ 15 & \text { H14 TS4 } & ++ \\ 16 & \text { H9 TS4 } & + \\ 17 & \text { R TS4 } & + \\ 18 & \text { Neg TS4 } & -\end{array}$

$$
\begin{gathered}
+++ \\
+++ \\
++ \\
++ \\
++ \\
++ \\
- \\
++ \\
++ \\
++ \\
- \\
++ \\
+ \\
+
\end{gathered}
$$$$
++
$$$$
++
$$$$
++
$$ 


\section{Discussion}

Vertical transmission of dengue viruses by Aedes aegypti has been reported in different geographic regions in the world. This study presented data and observation which confirmed the presence of transovarial transmission of dengue virus in Quezon City, Philippines, and that transovarial transmission may play an important role in initiating and maintaining the vector-borne disease in the locality.

The causative agent of the dengue disease is the dengue virus, a group of four flaviviruses that are closely related yet antigenically distinct. The four groups are known as serotypes and denoted as dengue virus type 1 (DEN-1), dengue virus type 2 (DEN-2), dengue virus type 3 (DEN-3) and dengue virus type 4 (DEN-4). The consistent estimation of infection in mosquitoes with arboviruses forms an important factor for surveillance and control. ${ }^{4}$

This current study tested the presence of intra-specific transmission through virus detection in adult stages of the mosquito, until F2 generation. Dengue virus (DEN-1, DEN2, DEN-3, DEN-4) was demonstrated to be positive in field adult mosquitoes in Quezon City and RITM samples through serotype-specific RT-PCR. This result may partially provide the reason why Quezon City is one of the dengue hotspots in the Philippines, ${ }^{10}$ it being one of the cities with high dengue cases recorded every year. The study results demonstrating transovarial transmission in two selected sites (H9 and H14) suggest that dengue viral infection in the area is not just primarily maintained via the Aedes aegyptihuman cycle or inter-specific transmission but via intraspecific transmission as well, up to the second generation. While it was shown in other studies that laboratory-infected mosquitoes can exhibit transovarial transmission up to the seventh generation, ${ }^{6,7}$ this is one of the first local papers to document this phenomenon in field-collected samples. In fact, in the subsequent offshoot studies of this research, vertical transmission was demonstrated to persist up to the $15^{\text {th }}$ generation..$^{15}$ Whether this persistence of dengue virus in the wild stock presents a potential and lingering threat has to be validated with virulence testing. Such experiment has been conducted for these samples and virulence was demonstrated in the mouse intracranial and intraperitoneal assays of the infected mosquito homogenates up to the F2 generation. ${ }^{16}$ What remains to be done is the virulence testing of infected mosquito homogenates up to the $15^{\text {th }}$ generation which this project is currently conducting.

The RITM (R-) mosquito samples were initially selected to establish a clean stock or dengue-free mosquitoes. However, results in this study showed that the R-samples were also positive for dengue. Further tests were conducted on the egg and the adult mosquitoes but these also showed similar positive results. Furthermore, the mosquito homogenates of these samples were also shown to exhibit virulence in the mouse intracranial and intraperitoneal assays. ${ }^{16}$

While earlier studies ${ }^{17}$ reported that the progeny of the mosquitoes infected with dengue virus do not show evidence of vertical infection by the virus, the results of this study suggest otherwise. In fact, a certain percentage of eggs in the succeeding generation transovarially transmit the dengue virus. A serotype-specific RT-PCR analysis of randomly collected mosquito eggs from a clutch showed a $20 \%$ transmission of dengue virus infection. ${ }^{14}$ Thus, transovarial transmission would be relative to the mosquito body (i.e., how the virus circulated and where it eventually colonized in the mosquito body) but in this case, it was documented to be present in the pooled samples up to the F2 progeny. This study provide the biological and molecular basis for such transovarial transmission of dengue virus that occurs in nature similar to those reported internationally. 3,6

Furthermore, while the data suggest direct transovarial transmission, there was a weakening of the viral presence noted, a typically expected event arising from cell dilution wherein, loss of detectable virus across generation occurred (i.e., RTS2, H9TS3 and RTS3). However, the data also suggest a possible recombination of sub-detectable viruses and reemergence at a later generation (i.e. H14TS1, H14TS4 and H14TS3). This phenomenon was also observed in a separate study involving field collected mosquitoes maintained as a colony up to 15th generation and could play as another important factor in the dengue viral persistence and seasonal virulence in the wild. ${ }^{14}$

\section{Conclusion}

The ecology and mechanisms of transovarial transmission of dengue virus in relation to the disease outbreak may not yet be fully explored especially in a country where dengue is dominant. However, the study findings presented evidences of occurrence of transovarial transmission of dengue virus by Aedes aegypti in the selected sites of Quezon City. Viral infection pattern of the progeny was observed until F2 generation. These observations show that transovarial transmission of dengue virus does occur in nature. The examination of dengue virus for field adult Aedes mosquitoes yielded positive results for all four (4) dengue serotypes until the F2 generation.

While vertical transmission may be responsible for the persistence of dengue virus in nature and eventually may play an important role in viral persistence in the wild stock, its current implications to the disease epidemiology, and up to some extent, on vector control (especially larval control), must be further studied. It is also important to consider genetic diversity among viruses within a serotype. Factors such as the biology and biting habits of the vector mosquitoes, retention of virulence on succeeding generations, environmental factors and immune responses 
of the population, and host susceptibility must also be put into consideration.

\section{Declaration}

The study was funded by the Philippines' Department of Science and Technology- Accelerated Science and Technology Human Resource Development Program (DOST-ASTHRD) through a thesis grant to Ralph Julius G. Bawalan. This research was also funded through a grant from the National Research Council of the Philippines (NRCP) through Francisco M. Heralde (NRCP Grant \# N001).

\section{Acknowledgments}

The authors recognize and thank the contributions of Quezon City Health Department particularly Dr. Antonieta Inumerable and Dr. Rolly Cruz; and the officials of Barangay Old Balara, Quezon City particularly Hon. Beda Torrecampo and Dra. Karen Alcid-See. Special thanks to Nurse Perlita Apelado for the assistance in serotype-specific RT-PCR analysis.

\section{References}

1. Hartanti MD, Suryani Tirtadjaja IA. Dengue virus transovarial transmission by Aedes aegypti. University Medicine. 2010; 29:65-70.

2. Akbar M, Ridad A, Djatie T, Kodyat S. PCR Detection of dengue transovarial transmissibility in Aedes aegypti in Bandung, Indonesia. Proc ASEAN Congr Trop Med Parasitol. 2008; 3:84-9.

3. Khin MM, Than KA. Transovarial transmission of dengue 2 virus by Aedes aegypti in nature. Am J Trop MedHyg. 1983; 32(3):590-4.

4. Chen WJ, Tsai SM, Chen SL, Ko YC, Fang AH. A study on transovarial transmission of dengue type 1 virus in Aedes aegypti. Zhonghua Min Guo Wei Sheng Wu Ji Mian Yi Xue Za Zhi. 1990; 23(4): 259-70.

5. Wasinpiyamongkol L, Throngrungkiat S, Jirakanjanakit N, Apiwathnasorn C. Susceptibility and transovarial transmission of dengue virus in Aedes aegypti: A preliminary study of morphological variations. Southeast Asian J Trop Med Public Health. 2003; 34 Suppl 2:131-5
6. Joshi V, Mourya DT, Sharma RC. Persistence of dengue-3 virus through transovarial transmission passage in successive generations of Aedes aegypti mosquitoes. Am J Trop Med Hyg. 2002; 67(2):158-61.

7. Throngrungkiat S, Maneekan P, Wasinpiyamongkol L, Prummongkol S Prospective field study of transovarial dengue-virus transmission by two different forms of Aedes aegypti in an urban area of Bangkok, Thailand. JVector Ecol. 2011; 36(1):147-52.

8. Lanciotti RS, Calisher CH, Gubler DJ, Chang GJ, Vorndam AV. Rapid detection and typing of dengue viruses from clinical samples by using reverse transcriptase-polymerase chain reaction. J Clin Microbiol. 1992; 30(3):545-51.

9. NSO. Census of Population. National Statistics Office [Online]. 2010 [cited 2013]. Available from http://www.nscb.gov.ph/activestats/psgc/ municipality.asp? muncode $=137404000 \&$ regcode $=13 \&$ provcode $=74$

10. DOH. Disease Surveillance Report. National Epidemiology Center Department of Health, Manila, Philippines [Online]. 2011 [cited 2012] Available http://www.doh.gov.ph/sites/default/files/2011Den14WMR.pdf.

11. DOH (2012). DOH- NEC Disease Surveillance Report of Dengue Case 2012 [Online]. [cited 2012 Oct]. Available from http://www.worldngayon.com/2012/06/dengue-surveillance-report2012/.

12. Mistica M, Delas Llagas L, Bertuso A. Dengue mosquito ovitrapping and preventive fogging trials in the Philippines. The Philippine Entomologist. 2008; 21(2):136-45.

13. Chan A, Chiang L, Hapuarachchi $\mathrm{H}$, et al. DNA barcoding. complementing morphological identification of mosquito species in Singapore. Parasites \& Vectors. 2014; 7:569.

14. Heralde FIIIM. Genomic Surveillance and Intervention on Dengue Virus in Mosquito. Proceedings of NRCP-Symposium on Dengue Researches. December 2014. The Bayleaf, Intramuros, Manila.

15. Obra G. Development of Sterile Insect Technique for Dengue Mosquito Vector, Aedes aegypti using Gamma Irradiation. Proceedings of NRCPSymposium on Dengue Researches. December 2014. The Bayleaf, Intramuros, Manila.

16. Bawalan R. Virulence of transovarially transmitted Dengue Virus in Aedes Mosquito. University of the East- Ramon Magsaysay (UERM) Memorial Medical Center, Inc. Graduate School. Quezon City, Philippines. 2014. Unpublished.

17. Angel B, Sharma K, Joshi V. Association of ovarian proteins with transovarial transmission of dengue viruses by Aedes mosquitoes in Rajasthan, India. Indian J Med Res. 2008; 128:320-3.

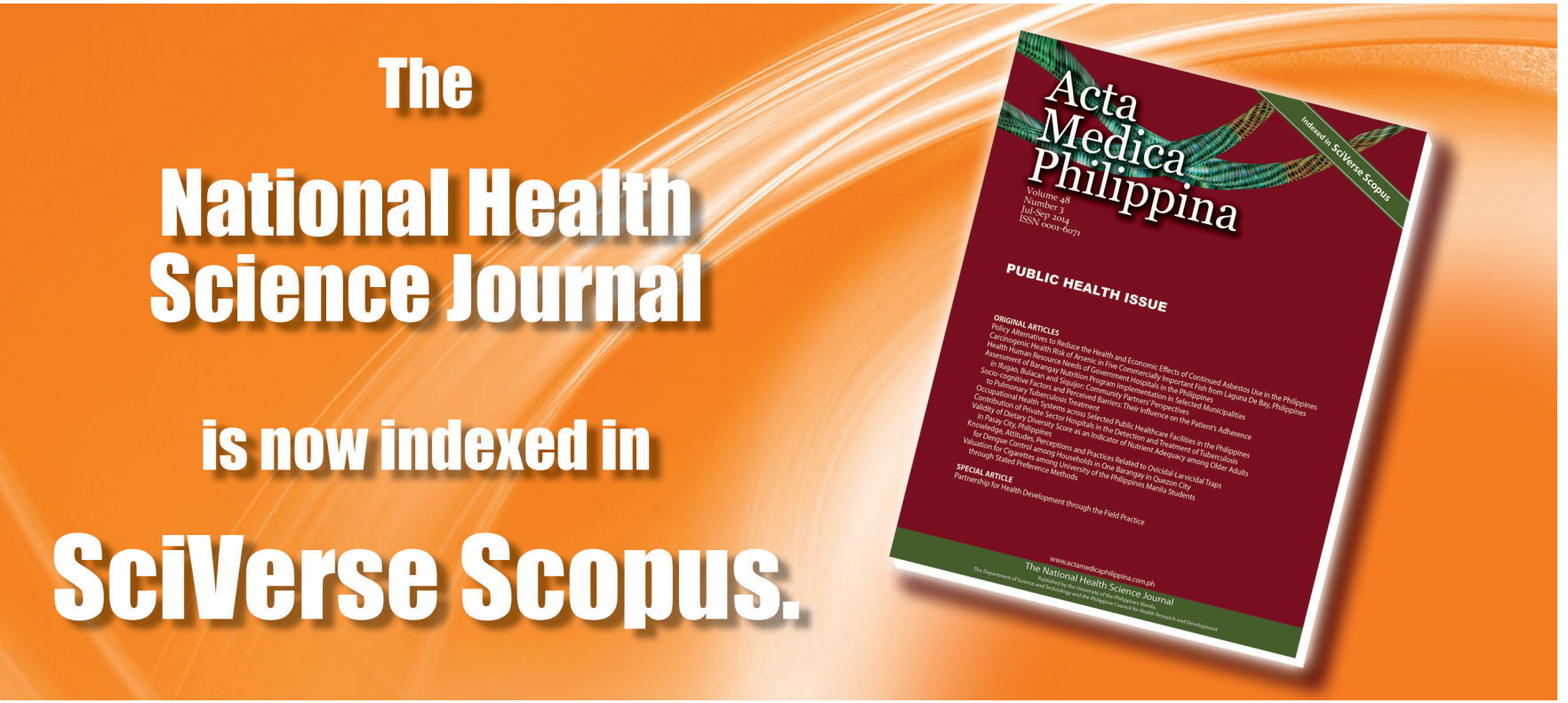

